\title{
Puzzling thermonuclear burst behaviour from the transient low-mass X-ray binary IGR J17473-2721
}

\author{
Jerome CHENEVEZ ${ }^{* \dagger}$ \\ DTU Space \\ E-mail: jerome@space.dtu.dk
}

\begin{abstract}
We investigate the thermonuclear bursting behaviour of IGR J17473-2721, an X-ray transient that in 2008 underwent a six month long outburst, starting (unusually) with an X-ray burst. We detected a total of 57 thermonuclear bursts throughout the outburst with AGILE, Swift, RXTE, and INTEGRAL. The wide range of inferred accretion rates (between $<1 \%$ and $\simeq 20 \%$ of the Eddington accretion rate $\dot{m}_{\text {Edd }}$ ) spanned during the outburst allows us to study changes in the nuclear burning processes and to identify up to seven different phases. The burst rate increased gradually with the accretion rate until it dropped (at a persistent flux corresponding to $\simeq 15 \%$ of $\dot{m}_{\text {Edd }}$ ) a few days before the outburst peak, after which bursts were not detected for a month. As the persistent emission subsequently decreased, the bursting activity resumed at a much lower rate than during the outburst rise. This hysteresis may arise from the thermal effect of the accretion on the surface nuclear burning processes, and the timescale is roughly consistent with that expected for the neutron star crust thermal response. On the other hand, an undetected "superburst", occurring within a data gap near the outburst peak, could have produced a similar quenching of burst activity.
\end{abstract}

8th INTEGRAL Workshop ?The Restless Gamma-ray Universe?

September 27-30 2010

Dublin Castle, Dublin, Ireland

\footnotetext{
* Speaker.

$\dagger$ On behalf of D. Altamirano, D. Galloway, J. in 't Zand, E. Kuulkers, N. Degenaar, M. Falanga, E. Del Monte, Y. Evangelista, M. Feroci, and E. Costa
} 


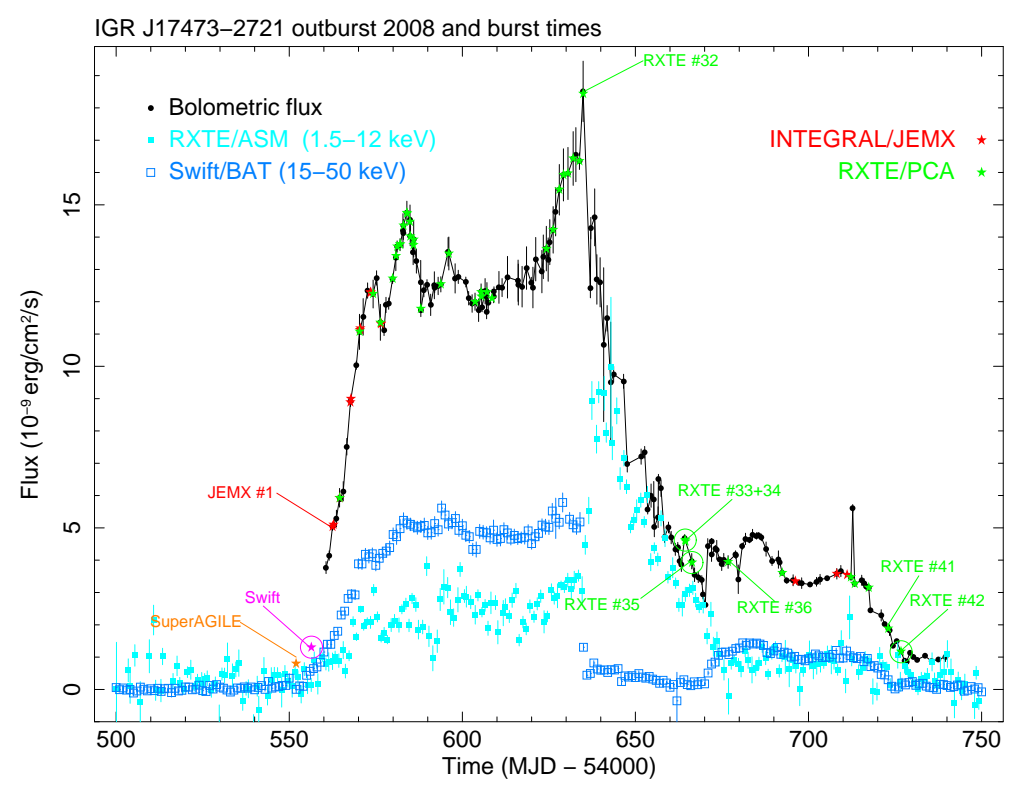

Figure 1: Broad band X-ray light curves for IGR J17473-2721 during the 2008 outburst, combining all available RXTE and Swift/BAT 1-day averaged data. The bolometric fluxes are derived from $R X T E /(P C A+H E X T E)$ fits and have been connected for clarity. Every PCA observation is represented by a black dot. The observed X-ray bursts are represented by stars, with circles for the four PRE ones, and those indicated by names delineate the transitions between different phases of burst behaviour .

\section{Introduction}

X-ray bursters are accreting neutron stars (NS) in low-mass X-ray binary (LMXB) systems, in which hydrogen $(\mathrm{H})$ and helium $(\mathrm{He})$ accumulates on the surface, periodically exploding in thermonuclear runaways. X-ray transients are ideal sources for studies of burst behaviour because they frequently experience a large range of accretion rates (and spectral states) in a short time frame. The X-ray transient source IGR J17473-2721 was discovered with the INTErnational Gamma-Ray Astrophysics Laboratory (INTEGRAL) in the Galactic Centre region during an outburst in April 2005 (Grebenev et al., 2005). The source exhibited a second episode of activity between March and September 2008, which was well followed from its beginning by most of the high energy satellite fleet operating at that time.

In the present paper, we report on the bursting behaviour of IGR J17473-2721 during its outburst in 2008. A more detailed study is available in (Chenevez et al., 2010).

\section{Burst behaviour as a function of the bolometric flux}

We found a total of 57 individual X-ray bursts from IGR J17473-2721 in 2008 (see Fig 1). The complete data set consists of 1 burst detected by SuperAGILE, 1 by Swift/XRT, 14 by INTEGRAL/JEM-X (of which 6 were also weakly detected by ISGRI), and 42 by RXTE/PCA.

During the rise phase of the outburst bolometric flux the burst recurrence time became progressively shorter, down to less than $2 \mathrm{~h}$, as derived from uninterrupted observations of the source between pairs of consecutive bursts. The light curves of the bursts observed in that phase of the 


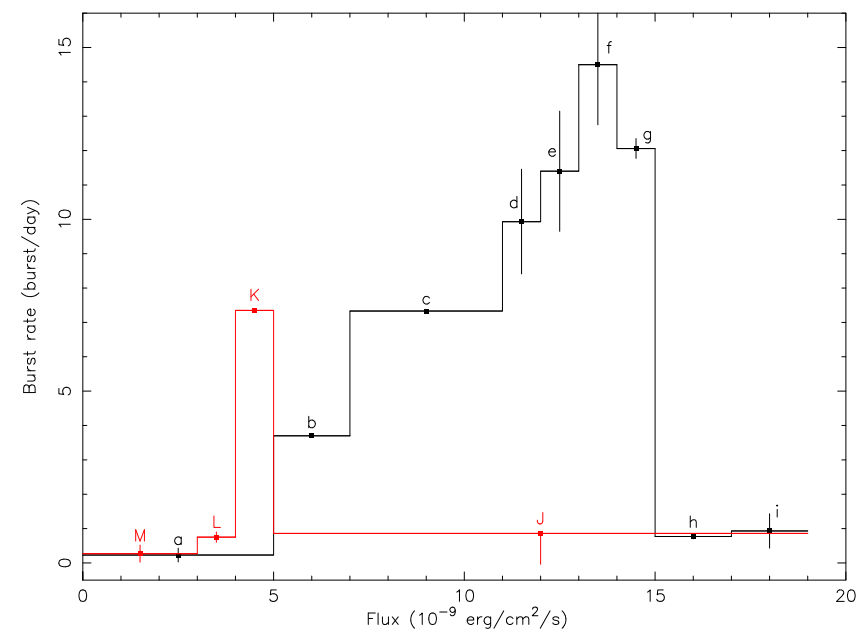

Figure 2: Plot of the IGR J17473-2721 burst rate as a function of persistent bolometric flux derived from the waiting times between two observed consecutive bursts. Bins in black (lower case letters) and red (upper case letters) correspond to pre- and post-outburst peak, respectively. The letters indicate the number of bursts $(n)$ and exposure times (exp in ks) per bin as follows: $n / \exp -\mathrm{a}: 2 / 11.2$, b: 3/39.8, c: 2/18.9, d: 8/50.6, e: 9/77.2, f: 8/44.1, g: 6/24.9, h: 5/16.8, i: 1/3.4, J: 0/60.2, K: 2/72.8, L: 9/55.1, and M: 2/40.9. The heights of the bins without error bars are given by one pair of bursts detected during an uninterrupted observation. The error bars are obtained either by the difference between the waiting times of two distinct pairs of bursts observed inside the same flux interval, or by the difference between the averaged time from burst to burst and the shortest time within two consecutive burst detections in separated observations.

outburst showed slow rise and relatively long decay times. After the persistent flux reached its maximum, corresponding to $\simeq 15-20 \%$ of the Eddington limit (see Fig. 1), no bursts were detected during a prolonged period of low flux in a soft spectral state. The burst activity resumed after an interruption of one month, with shorter and more intense bursts than before the peak of the outburst.

We adopt here the bolometric $(0.1-200 \mathrm{keV})$ flux as a tracer of the accretion rate. The burst rate as a function of persistent emission (or accretion rate) plotted in Fig. 2 indicates a steady increase until a maximum of 14.5 bursts per day is reached at a bolometric flux of $\approx$ $13.5 \times 10^{-9} \mathrm{erg} \mathrm{cm}^{-2} \mathrm{~s}^{-1}$. The red histogram (labeled with uppercase letters) of Fig. 2 displays the burst rate during the decrease of the persistent emission. Indeed, no bursts at all were detected in the 30 days following the bolometric peak flux of the outburst, even though observations totalling approximately $80 \mathrm{ks}$ were made with RXTE. Before the burst interruption, JEM-X and PCA detected 42 bursts in a total of about $280 \mathrm{ks}$ exposure time, corresponding to an average burst rate of almost 13 bursts per day. The burst activity thus resumed about one month after the outburst peak and decreased later on with the decreasing persistent emission. So, for the same range of bolometric flux, the bursting activity in IGR J17473-2721 strongly depends on whether the persistent emission is increasing or decreasing. Moreover, we note from Fig. 2 that, apart from the two bursts observed by SuperAGILE and Swift at the beginning of the outburst, the bursts observed during the rise phase of the outburst occurred at a persistent bolometric flux above $5 \times 10^{-9} \mathrm{erg} \mathrm{cm}^{-2} \mathrm{~s}^{-1}$, while all the bursts observed during the outburst decrease phase occurred below this threshold. 


\section{Discussion}

\subsection{Summary of main results}

Thanks to the wide coverage by RXTE, INTEGRAL, Swift and AGILE and their 58 burst detections, the 6-month long outburst from the transient IGR J17473-2721 provides a comprehensive record of burst activity on a neutron star over a wide range of accretion rates. The most interesting aspect of this record is the hysteresis shown in Fig. 2, particularly the 1-month long intermission in burst activity that commences with the peak of the accretion outburst and at the same time of a spectral state change in the accretion radiation.

From the observation of PRE bursts (see Chenevez et al., 2010) we derive the distance to the source at $\simeq 5.4 \mathrm{kpc}$ by assuming the highest burst peak flux equal to the Eddington limit for a H-poor photosphere: $L_{\mathrm{Edd}}=3.8 \times 10^{38} \mathrm{erg} \mathrm{s}^{-1}$ (Kuulkers et al., 2003).

In summary, the originality with IGR J17473-2721 is that seven phases of burst behaviour can be distinguished in Fig. 1:

1. Very early in the outburst, a first burst detected in hard X-rays with SuperAGILE, followed a few days later by the Swift observation of a PRE burst. Both bursts occur at a low accretion rate $\lesssim 1 \%$ of the Eddington limit;

2. During the rise of the outburst and the subsequent plateau phase in hard state (from the first JEM-X burst to RXTE \#24), bursts arrive faster with increasing accretion rates. The slope of the linear rise in the burst rate versus persistent flux histogram (Fig. 2) is equivalent to $\alpha=80 \pm 16$. Together with this value, the relatively long burst rises and decays point to $\mathrm{He}$-ignited $\mathrm{H} / \mathrm{He}$ bursts in which the $\mathrm{H}$ is burnt through the rp process;

3. During the final $\simeq 15$ days leading to the outburst peak (RXTE bursts \#25 to \#32), when the bolometric flux is in excess of $15 \times 10^{-9} \mathrm{erg} \mathrm{s}^{-1} \mathrm{~cm}^{-2}$, the recurrence time drops by an order of magnitude (Fig. 2). This leads to an increase of alpha about 1500, consistent with simultaneous stable and unstable He burning;

4. At the time of the outburst peak, a 1-month intermission in burst activity starts. The outburst peak also coincides with a sharp spectral transition into a soft state;

5. After the intermission, when the outburst is still in the soft state, but when the persistent flux is at similar levels as during the first few bursts early in the outburst, the brightest and shortest X-ray bursts occur (RXTE bursts \#33-35, two with PRE). Most likely these are pure He flashes;

6. After the persistent spectrum switches back to a hard state within $10 \mathrm{~d}$, the X-ray bursts become longer again, but not as long as earlier in the outburst, and are probably again due to mixed He/H burning (JEM-X bursts \#11-14 and RXTE bursts \#36-41);

7. the last observed burst ( $R X T E \# 42$ ) occurs after yet another transition of the accretion radiation from a hard to a soft state at low accretion rate. This burst also shows limited PRE. 


\subsection{Possible interpretations}

There is considerable variability in burst peak fluxes and time scales. The rise and decay times tend to be somewhat longer before the intermission than after, particularly the rise times. The shortest rise times combine with the highest peak fluxes. These trends indicate a varying $\mathrm{He}$ to $\mathrm{H}$ abundance ratio in the burning layer. This is not unexpected considering the varying accretion rate. The shortest bursts (RXTE bursts \#33-35) are consistent with pure He burning. All other bursts are longer and fainter and must have larger $\mathrm{H}$ fractions in the fuel. Therefore, the mass donor must be $\mathrm{H}$-rich. The fact that pure $\mathrm{He}$ bursts occur implies that stable $\mathrm{H}$ burning (through the hot CNO cycle) must simultaneously be present during RXTE bursts \#33-35, particularly since bursts at similar persistent fluxes early in the outburst (for instance RXTE burst \#1) have roughly twice longer times scales and half the peak flux. Thus, we identify four burst regimes. First, mixed H/He flashes during burst phases i, ii, iii and vi. Second, pure He flashes concurrent with stable $\mathrm{H}$ burning in burst phase v. Thirdly, rich He flashes at the lowest persistent fluxes. Lastly, no bursts at all and, therefore, stable $\mathrm{H}$ and $\mathrm{He}$ burning during the intermission. Thus, all burst regimes for $\mathrm{H} / \mathrm{He}$ burning (e.g. Fujimoto et al., 1981) are exhibited by IGR J17473-2721, but at accretion rates about one magnitude order higher.

The most intriguing question is what actually caused the 1-month interruption of burst activity after the peak of the outburst. If the $\mathrm{He}$ and $\mathrm{H}$ are not burnt in an unstable fashion through flashes, they must be burnt in a stable fashion. The drop in burst rate (Fig. 2) just before the peak thus suggests the onset of stable $\mathrm{H}$ and $\mathrm{He}$ burning, at a threshold persistent luminosity that is similar (i.e., within a factor of two) to what is seen in other bursters, although the burst profiles in IGR J174732721 do not show as strong a change. Hence, stable He burning, which should occur at higher accretion rates than stable H burning, apparently starts for IGR J17473-2721 at lower accretion rates than normally expected. We discuss two scenarios.

One possibility is that the intermission is the result of thermal relaxation of the crust. The crust becomes hotter during the outburst due to electron capture reactions. The thermal time scale of the crust is of order a few months. It is likely that the temperature is still rising after the outburst peak. Above certain temperatures $\left(7 \times 10^{7}\right.$ and $4 \times 10^{8} \mathrm{~K}$, respectively) the $\mathrm{H}$ and $\mathrm{He}$ burning will become stable. The intermission of bursts at the time of the peak of the outburst may be explained if the temperature rises above the threshold temperature for stable He burning just at that time. The thermal relaxation of the crust may sustain that temperature for the duration of the intermission after which it would drop below the threshold due to the decay in accretion rate that started at the peak of the outburst. A problem of this scenario is that the heating time scale appears short if the burning depth is the same for stable H and stable He burning. Indeed, it takes for IGR J17473-2721 less than 15 days from the onset of stable $\mathrm{H}$ burning (drop of burst rate) to reach stable-only He burning (burst interruption), corresponding to a difference factor of 5-6 in temperature. The rapidity of this heating may be explained by the onset of stable $\mathrm{H}$ burning, but verifying this requires solving the heat balance of the upper layers. As with the calculation of the thermal relaxation of the crust, this is outside the scope of this observational paper.

An alternative and perhaps more likely explanation for the intermission is that a superburst occurred during a data gap, and provided the sudden heating to initiate stable He burning. Indeed, there is a data gap of more than 10 hours between the last detected burst and the first available ob- 
servation (by RXTE/ASM) of the soft state. Though the probability that a superburst just occurred during the data gap is rather small $-\sim 0.1 \%$ for a typical superburst recurrence time of $\sim 1$ per year - this is, in principle, enough time for a superburst to ignite and cool off. Cessation of burst activity after a superburst is commonly observed. This 'quenching' of normal bursts is explained by the additional heat flux coming from the underlying cooling ashes of the superburst that stabilizes the $\mathrm{H} / \mathrm{He}$ burning layers. A similar example to the present case, is a superburst from the transient and H-rich burster 4U 1608-52 (Keek et al. 2008), 55 days after the onset of an outburst in 2005. The first normal burst was detected 100 days after the superburst, although long data gaps may have prevented earlier burst detections. Considering that the sudden burst intermission of IGR J174732721 began after the outburst peak, a superburst would thus possibly have taken place more than 75 days after the outburst onset. The fluence of the outburst until the peak is $0.08 \mathrm{erg} \mathrm{cm}^{-2}$, which at the distance to the source translates to an energy of $3 \times 10^{44} \mathrm{erg}$. This is similar to the value reported by Keek et al. (2008) before the superburst of $4 \mathrm{U}$ 1608-522. The average bolometric flux of the outburst prior to the maximum is $12.3 \times 10^{-9} \mathrm{erg} \mathrm{cm}^{-2} \mathrm{~s}^{-1}$ or about $12 \%$ of the Eddington limit, which corresponds to the level of accretion rate typically measured for superbursts. For $\Delta t=$ 75 days, this corresponds to an accumulated column depth $y=m \Delta t /(1+z)=1.1 \times 10^{1} \mathrm{~g} \mathrm{~cm}^{-2}$. This is one order of magnitude below the typical column depth for superbursts whose energy release is $10^{42} \mathrm{erg}$, but a part of the necessary $\mathrm{C}$ fuel may have been accumulated on a much longer timescale at low accretion rate. As previously mentioned, both stable and unstable He burning likely occured during the 15 days prior to the outburst peak of IGR J17473-2721, thus increasing considerably the $\mathrm{C}$ production.

In conclusion, the burst properties of IGR J17473-2721 are consistent with a NS accreting Hrich material at varying rates all along the outburst episode. The bursting rate as a function of the accretion rate displays an hysteresis on either side of the peak of the outburst, coincident with the interruption of the burst activity. This hysteresis may be explained by the thermal response of the NS crust, or the occurrence of a superburst. Though the odds of having missed a superburst inside a data gap of only 10 hours are not high, we consider that explanation as more likely than thermal relaxation of the crust. The question is whether the occurrence of this undetected superburst is related to the abrupt spectral transition the source underwent short after the peak of the outburst.

\section{Acknowledgements}

JC acknowledges financial support from IDA and ESA/PRODEX Nr. 90057.

\section{References}

Chenevez J., Altamirano D., Galloway D.K., in 't Zand J.J.M., et al., 2010, MNRAS, in press

Fujimoto M.Y., Hanawa T., and Miyaji S., 1981, ApJ, 247, 267

Grebenev S.A., Molkov S.V., and Sunyaev R.A., 2005, ATel 467

Keek L., in 't Zand J., Kuulkers E., et al., 2008, A\&A, 479, 177

Kuulkers E., et al., 2003, A\&A, 399, 663 